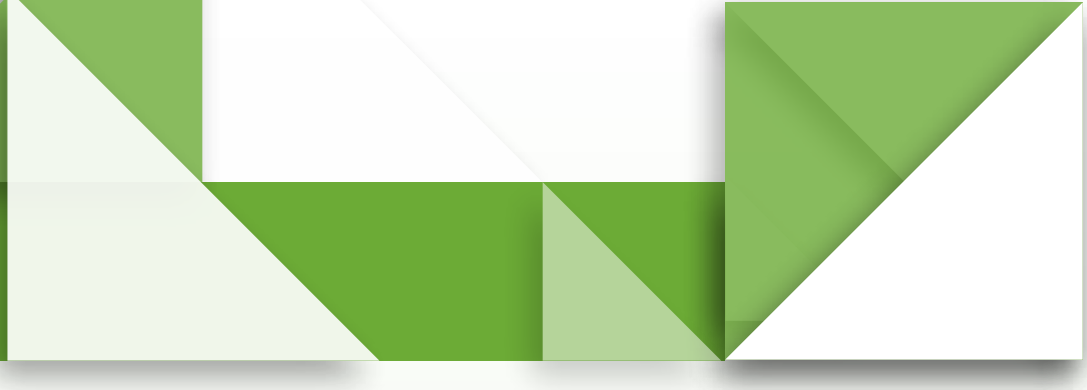

\title{
Pensamiento crítico dominio- específico en la didáctica de las ciencias
}

\section{- Critical thinking as specific domain in sciences didactics \\ - Pensamento crítico domínio-específico na didática das ciências}

\section{Resumen}

En primera instancia se hace una discusión general acerca del objeto de estudio de la didáctica de las ciencias y su relación con el campo de la pedagogía; esta lleva a establecer algunos límites y tensiones entre la formación y el pensamiento crítico en dominios específicos del conocimiento, como eje central de la didáctica de las ciencias, el cual desborda los convencionales propósitos centrados en la enseñanza y en el aprendizaje de las ciencias. A lo largo del texto se privilegia la tesis del desarrollo del pensamiento crítico de dominio específico, lo cual nos lleva, en segundo lugar, a presentar resultados de investigación sobre la expresión del pensamiento crítico en niños, niñas y maestros de grados cuarto y quinto de educación básica primaria de 56 instituciones públicas de la ciudad de Manizales, en el campo de las ciencias naturales. El estudio fue de naturaleza mixta y las categorías investigadas fueron solución de problemas, metacognición y argumentación, las cuales se consideran constituyentes del pensamiento crítico en los estudiantes. Para la recolección de la información se diseñaron diez talleres en los cuales se presentaban situaciones problema sobre diferentes temáticas de ciencias. Los análisis se realizaron con 224 niños y 5 maestros. El estudio se adelantó en tres momentos, en cada uno de los cuales se realizó el análisis de cada una de las categorías, así como el análisis de las relaciones entre ellas. Se presentan los resultados independientes para cada una de las tres categorías investigadas, así como un análisis comprensivo de la relación entre ellas en función de comprender la expresión del pensamiento crítico en los niños investigados. Se describieron comprensivamente las concepciones de los maestros acerca del pensamiento crítico.

\section{Óscar Eugenio Tamayo Alzate*}

Universidad de Caldas-Universidad Autónoma de Manizales.oscar.tamayo@ucaldas.edu.co

\section{Palabras clave:}

Didáctica,

ciencias,

enseñanza,

aprendizaje,

pensamiento

crítico. 


\section{Abstract}

It is the purpose of this paper to briefly discuss the object of study in Sciences Didactics and its relation to pedagogy, so that, teachers can establish some limits and tensions between education and critical thinking in specific domains of knowledge, which represent bases for science learning and teaching. The study proposes critical thinking as a learned skill that needs instruction and practice in a specific domain and presents research results of a study regarding elementary students and teachers critical thinking skills in 56 public schools from Manizales. The categories taken into consideration for this study were: problem solving, metacognition and argumentation, because they are considered the core to start the critical thinking development. Data was collected by using ten workshops which presented problematic situations regarding different sciences topics. The participants were 224 children and five teachers who actively worked in three different moments that were intended to develop the three proposed categories as well their relations. The results were analyzed for each of the three categories and a comprehensive analysis was done to establish the relationship between them so that we can understand the critical thinking development in the participants. Finally, teachers and students conceptions of critical thinking are described within the context of science education.

\section{Resumo}

Em uma primeira instância faz-se uma discussão geral acerca do objeto de estudo da didática das ciências e sua relação com o campo da pedagogia. Esta discussão leva a estabelecer alguns limites e tensões entre a formação e o pensamento crítico em domínios específicos do conhecimento, como eixo central da didática das ciências, indo além dos objetivos convencionais centrados no ensino e aprendizagem das ciências. Ao longo do texto se privilegia a tese do desenvolvimento do pensamento crítico de domínio específico, o qual nos leva, em segundo plano, a apresentar resultados de investigação sobre a expressão do pensamento crítico em meninos, meninas e professores da quarta e quinta séries da educação básica de 56 instituições públicas da cidade de Manizales, no campo das ciências naturais. $O$ estudo foi de natureza mista e as categorias investigadas foram resolução de problemas, metacognição e argumentação, as quais são consideradas constituintes do pensamento crítico dos estudantes. Para a constituição dos dados, foram planejadas dez oficinas nas quais se apresentavam situações-problema sobre diferentes temas das ciências. As análises se realizaram com 224 crianças e 5 professores. $O$ estudo se dividiu em três momentos, em cada um dos quais se realizou a análise de cada uma das categorias, assim como a análise das relações entre elas. São apresentados os resultados independentes para cada uma das três categorias investigadas, assim como uma análise relacionada à relação entre elas em função da compreensão da expressão do pensamento crítico nas crianças investigadas. São descritas, ainda, as compreensões sobre as concepções dos professores acerca do pensamento crítico.

\section{Key words:}

Teaching, science, learning, critical thinking.
Palavras chave:

Didática, ciências, ensino, aprendizagem, pensamento crítico. 


\section{Desarrollo de la propuesta}

La didáctica de las ciencias en la actualidad constituye un campo del saber en el que confluyen reflexiones y aportes de diferente naturaleza provenientes de muy diversos campos del conocimiento, entre los cuales históricamente se han destacado la filosofía, la psicología y la pedagogía. Estas áreas son, en gran parte, orientadoras de desarrollos futuros en el ámbito de la didáctica y señalan ciertas tensiones en virtud de los logros en cada uno de ellos y en la didáctica de las ciencias.

Además de los valiosos aportes de los tres campos ya mencionados a la didáctica de las ciencias, en años más recientes vemos fuertes influencias que proponen nuevos desafíos a los profesores, tanto por los requerimientos conceptuales que demandan, como por las posibilidades de incorporarlos de manera práctica en las actividades cotidianas del aula de clase. A manera de ilustración, son valiosas las contribuciones de los estudios del lenguaje a la didáctica en cuanto a los análisis del discurso y del contenido en el aula y su relación con: el aprendizaje de los conceptos y teorías, el manejo del poder en al aula, el análisis de la comunicación entre estudiantes, profesores/ estudiantes, estudiantes/estudiantes, entre otras. De especial importancia en la actualidad son las investigaciones orientadas a desarrollar en los estudiantes habilidades argumentativas (Erduran 2008, Jiménez-Alexandre, 2008, Tamayo, Zona y Loaiza, 2014; Ruiz, Márquez y Tamayo, 2014), y el estudio de las analogías (Dagher, 1994, Justi, 2006), metáforas (Palma, 2004) y múltiples representaciones en la enseñanza de las ciencias (Tamayo et al., 2011).

Los aspectos esbozados hasta el momento no nos deben llevar a pensar que el campo de la didáctica está constituido solo por el aporte de otras áreas disciplinares, a manera de una ciencia que se construye a partir de los avances de las otras. Parece claro hoy que la didáctica ha redefinido su objeto de estudio en función de los procesos de enseñanza, aprendizaje y formación de pensamiento crítico en dominios específicos del conocimiento, objetos que llevan a reconocer nuevos problemas de investigación, con sus consecuentes desarrollos metodológicos que hacen posible su estudio y diferentes comunidades académicas dedicadas de manera específica a estos nuevos problemas (Toulmin, 1972; Porlán, Rivero y Martín del Pozo, 1998).

\section{Tensiones entre la pedagogía y la didáctica de las ciencias}

Con el propósito de precisar usos terminológicos, proponemos la formación como el objeto de estudio de la pedagogía; de igual manera, consideramos la educación como el campo general en el cual se inscriben tanto la pedagogía como la didáctica. Dada la intencionalidad de este documento, dedicaremos algunas líneas a profundizar solo en las interacciones de orden conceptual que se pueden tejer entre pedagogía y didáctica con el único interés de delimitar sus objetos de estudio.

Por lo general, se ha considerado a la didáctica de las ciencias como un saber de naturaleza instrumental inherente a la pedagogía (figura 1a). Ha sido un saber centrado en responder al problema de cómo enseñar en las aulas de clase, y en el que sus desarrollos se restringen de manera extrema a saberes de orden procedimental. Desde esta perspectiva las preguntas, ya clásicas, que se hacen los maestros exploran por las formas de enseñar, las secuencias de enseñanza, las maneras de evaluar, de mantener la disciplina en el aula, etc. En esta línea de pensamiento, la didáctica se constituye en un cómo, en el que los principios teóricos que lo determinan provienen 
de la pedagogía. Es decir, no se le reconoce a la didáctica un lugar conceptual que no sea aquel de determina acciones especificas en cuanto a la enseñanza, diríamos en términos generales una teoría sobre la acción práctica.

Otros tipos de relación entre pedagogía y didáctica las ilustramos en la figura 1b. La pedagogía y la didáctica pueden tener en común: problemas específicos, conceptos bisagra, marcos teóricos, desarrollos metodológicos, intencionalidades, propósitos, espacios, lugares, poblaciones objeto de estudio, etc. Estos puntos de encuentro permitirían de alguna manera el trabajo integrado en función del estudio de problemáticas comunes en los que los aportes desde cada uno de ellos pueden aportar a su solución. En este modelo de interacción pedagogía/ didáctica (P/D), existirían regiones difusas en las que los objetos de estudio de una y otra estarían imbricados, asimismo habría regiones más diferenciadas en las que se identificarían con mayor precisión desarrollos teóricos y metodológicos propios de cada uno de estos dos campos disciplinares.

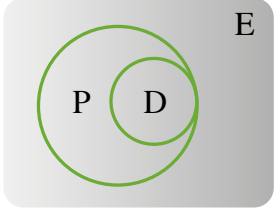

a

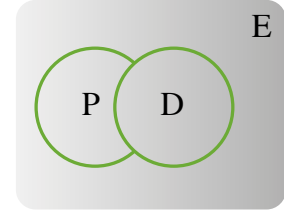

b

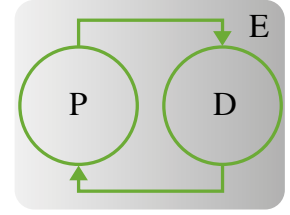

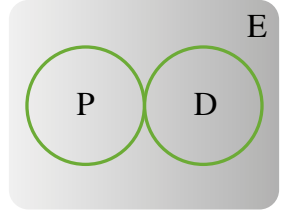

d

Figura 1. Diferentes modelos de interacción entre pedagogía y didáctica. P: pedagogía;

D: didáctica; E: educación.

Si continuamos en el ejercicio de diferenciar los campos conceptuales de la pedagogía y la didáctica llegamos al modelo c (figura 1c) en el que los objetos de estudio de una y otra están bien definidos. Aquí el propósito central de la pedagogía estaría en función de generar modelos abstractos en torno a la formación de niños, niñas, jóvenes, adultos, formación para la paz, para la ciudadanía y, en síntesis, aportar a la formación holística de sujetos y comunidades. De otra parte, desde el lugar de la didáctica, su objeto de estudio estaría centrado en la cualificación de los procesos de formación disciplinar en los diferentes niveles de la educación. En este caso, a diferencia del discurso de la pedagogía, el de la didáctica integra la dimensión abstracta inherente a su campo, con la dimensión práctica vivida en el oficio de ser maestro. La existencia de fuerzas, tipo interacciones gravitacionales, entre pedagogía y didáctica, hace imposible su separación total, de tal manera que la una y la otra orbitan mutuamente y aportan desde sus propios lugares, y desde las interacciones que desde ellas se establezcan, a la solución de problemas. En este modelo de interacción son viables los desarrollos teóricos y metodológicos de manera autónoma tanto de la pedagogía como de la didáctica.

Si avanzamos un paso más en este proceso de diferenciación, y apoyados en estudios bibliométricos (Pfundt y Duit, 1991, 1993), vemos cómo se vienen 
consolidando las didácticas de dominio específico, con la construcción de marcos teóricos coherentes y consistentes y con desarrollos metodológicos particulares y específicos (figura 1d). Asimismo, observamos un mayor distanciamiento de los saberes propios de la pedagogía en torno a la formación y un interés centrado en el logro de explicaciones, comprensiones y transformaciones en el marco de los procesos de formación en campos específicos del conocimiento. Este distanciamiento entre pedagogía y didáctica demanda de cada uno de estos dos campos la definición y delimitación precisa de sus objetos de estudio, así como de sus plataformas metodológicas coherentes y consistentes con tales objetos de estudio.

No discutiremos aquí las ventajas o desventajas de asumir uno u otro modelo de interacción entre pedagogía y didáctica, sin duda este es un ejercicio académico valioso que puede aportar herramientas conceptuales y metodológicas para el trabajo en el aula de clase. Nuestro propósito central en este aparte es mostrar algunas tensiones que en la actualidad se dan entre pedagogía y didáctica, producto, sin dudas, de los desarrollos internos que se ha dado en cada uno de estos campos durante las últimas décadas, especialmente los provenientes de la didáctica general y de las didácticas de dominio específico.

Al igual que mostramos ciertas tensiones entre los campos de la pedagogía y la didáctica, podemos señalar, asimismo, cierta diferenciación entre los objetos de estudio de la didáctica general y los de las didácticas de dominio específico (figura 2).

Proponemos el estudio de la didáctica general a partir de la confluencia de seis campos con desarrollos teóricos y metodológicos aplicables a la enseñanza y aprendizaje de: las Ciencias Sociales, las Ciencias Naturales,

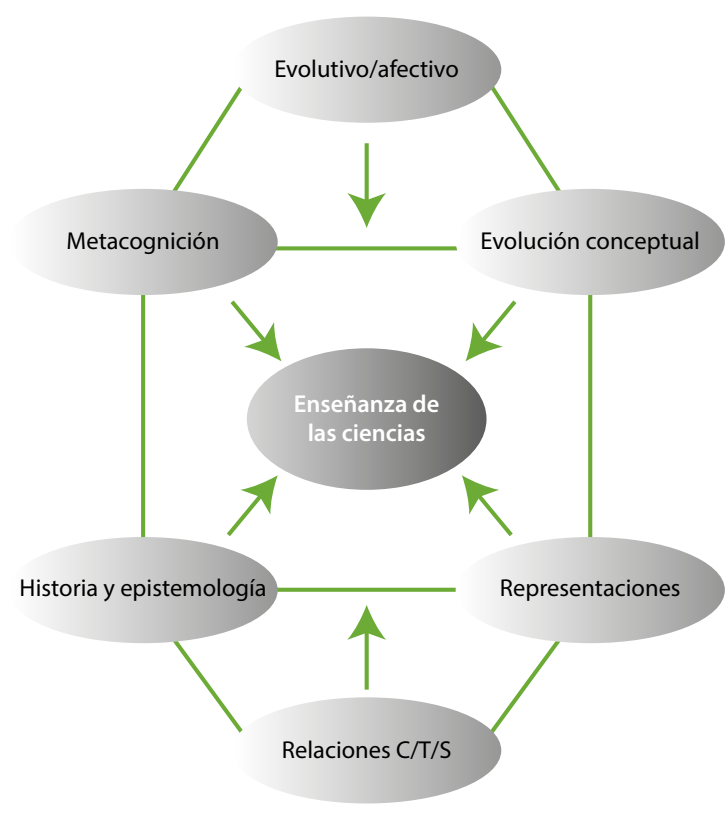

Figura 2. Campos que aportan a la construcción de un nuevo concepto de didáctica general.

las Ciencias Humanas y las Artes. Estas áreas son: representaciones, historia y epistemología, relaciones ciencia/tecnología/sociedad/ ambiente $(C / T / S / A)$, emociones, aprendizaje como evolución y metacognición (figura 4). Los desarrollos en cada uno de estos campos transgreden los límites propios de las didácticas de dominio específico, así hayan surgido en el seno específico de una de ellas y pasan a consolidar un cuerpo de conocimientos común a todas ellas.

\section{Tensiones entre la didáctica general y las didácticas de dominio específico}

Si bien defendemos la existencia de desarrollos teóricos y metodológicos comunes a las didácticas de diferentes campos del saber, también es claro que reconocemos los desarrollos propios que se generan en las múltiples interacciones entre las ciencias de referencia (Matemáticas, Química, Sociales...), y su didáctica específica (figura 3). A manera de ilustración, la enseñanza de la química en un 
contexto específico -en el cual los profesores que la enseñan y los estudiantes que la aprenden ponen en juego sus experiencias, intereses y motivaciones-, puede lograr nuevos desarrollos no contemplados en el marco de la didáctica general. En otras palabras, las didácticas de dominio específico no se limitan a la aplicación de aquellos principios teóricos y metodológicos provenientes de la didáctica general; por el contrario, se constituyen en una fuente inagotable de experiencias específicas que parten del reconocimiento de la naturaleza de la ciencia a enseñar y de las especificidades contextuales en las que se realiza el proceso de formación; son, en gran parte, el laboratorio en el cual se generan nuevas experiencias de enseñanza, de aprendizaje y de formación en pensamiento crítico disciplinar y, como tal, sus logros permean de manera permanente los marcos conceptuales y metodológicos ya constituidos de la didáctica general.

Los aportes de las didácticas específicas a la didáctica general y a la pedagogía parten del estudio de problemáticas específicas, tal es el caso de los recientes hallazgos de la argumentación en ciencias, los procesos de autorregulación en el aprendizaje de la lectura, el empleo de las múltiples representaciones en la enseñanza y aprendizaje de las matemáticas, de los aportes de la visualización en el aprendizaje de la química, entre muchos otros (figura 3).

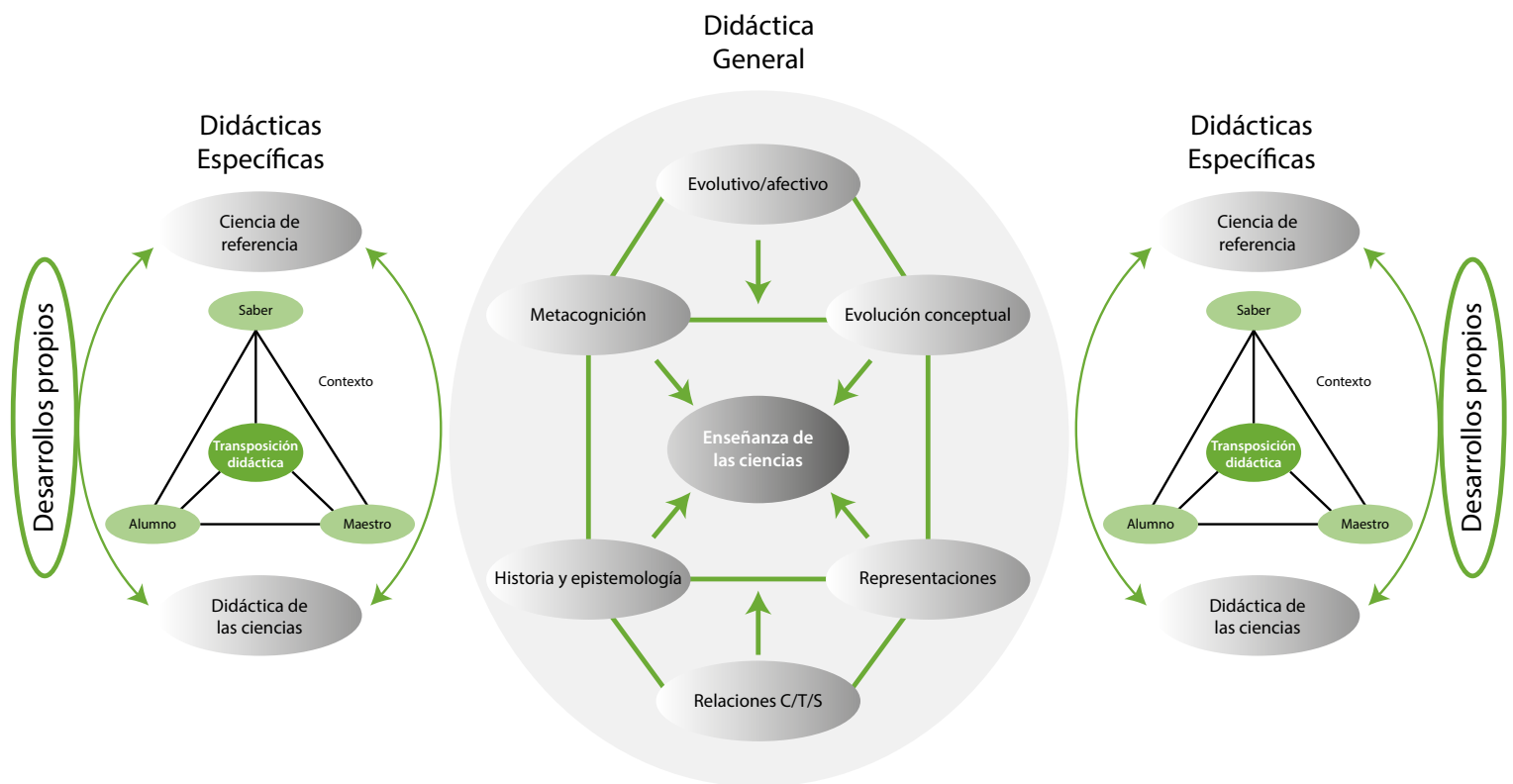

Figura 3. Interacciones entre didáctica general y didácticas de dominio específico.

Los desarrollos logrados en el marco de una didáctica específica eventualmente entran a constituirse en aportes para otras didácticas; sin embargo, es claro que llegar a ser de utilidad para otros campos de la enseñanza exige procesos de validación que requieren tanto del juicio crítico de maestros e investigadores como de tiempos que suelen ser exageradamente largos si los comparamos con aquellos empleados en otros campos como las ciencias de la salud y las ingenierías. 


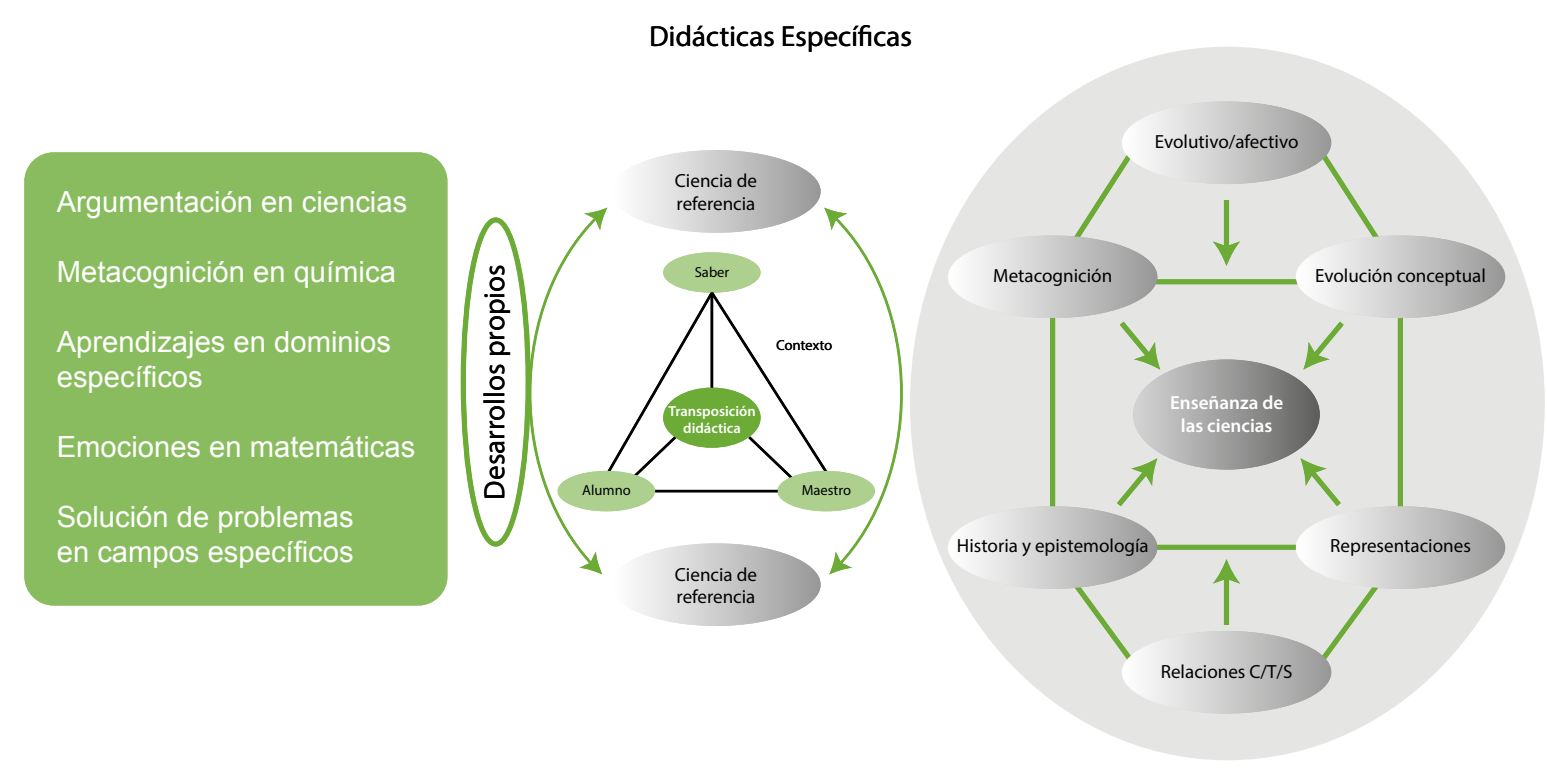

Figura 4. Origen de nuevos problemas de investigación en el interior de las didácticas de dominio específico.

Las relaciones entre la didáctica general y las didácticas de dominio específico son, sin duda, de naturaleza más cercana que aquellas entre la pedagogía y la didáctica. En consecuencia, los procesos de transferencia de saberes de unas didácticas dominio-específicas a otras pueden ser más ágiles y requerir ajustes menores que los necesarios para la transferencia de saberes educativos y pedagógicos al ámbito de la didáctica o viceversa.

\section{La didáctica de las ciencias y la formación de pensamiento crítico dominio-específico. Algunos resultados de investigación}

Uno de los propósitos centrales de la educación en los diferentes niveles educativos es aportar a la formación de pensamiento crítico en los estudiantes, aspecto sobre el cual existen diversas perspectivas teóricas y metodológicas para su estudio (Tamayo, Zona y Loaiza, 2014). En términos generales, las perspectivas de estudio que más han influido históricamente han incorporado discusiones filosóficas, psicológicas, educativas y sociales, cada una de ellas con autores representativos. Asimismo, encontramos en la actualidad desarrollos que amalgaman las distintas vertientes antes señaladas, así como orientaciones que empiezan a constituirse valiosas en función de vincular el desarrollo del pensamiento crítico con los dominios específicos del conocimiento.

La reflexión sobre el pensamiento crítico surgió en la filosofía e históricamente ha tenido aportes valiosos desde la psicología. En términos de Vélez (2013, p. 34) "en la actualidad es un objeto de estudio de distintos campos disciplinares, en particular de la Psicología, la Sociología Política, la Pedagogía y, más recientemente, la Didáctica de las Ciencias". Continúa el autor planteando que "... las particularidades de nuestro contexto latinoamericano (y colombiano), tal vez, hacen más pertinente asumir una posición integradora, interdisciplinar, que permita articular las dinámicas de formación y desarrollo científico y tecnológico con los grandes conflictos sociales, políticos e ideológicos que coexisten en nuestra región. Esto significa que podemos transformar nuestras dinámicas educativas e 
investigativas, utilizando el pensamiento crítico como herramienta conceptual y metodológica, como recurso intelectual si se quiere, sin perder de vista un horizonte más complejo e importante: la transformación de nuestras condiciones de injusticia y desigualdad social. En síntesis, el desarrollo del pensamiento crítico se puede convertir en una estrategia para la emancipación individual y colectiva, en la que son imprescindibles los procesos educativos y la producción de información y de conocimiento" (Vélez, 2013: p 34-35).

Son muchas las perspectivas teóricas desde las cuales se conceptualiza el pensamiento crítico, tal es el caso de aquellas centradas en el desarrollo de capacidades, en competencias, en habilidades, en disposiciones y en criterios, entre otras. Independientemente del lugar conceptual desde el cual se considere el pensamiento crítico, se requiere que el sistema educativo, como un todo, oriente esfuerzos en función de lograr su formación. En este sentido, el actuar de maestras y maestros en las aulas, en las instituciones educativas y en los diferentes contextos formadores, se vuelve esencial en función, no solo de aportar a la formación integral de los estudiantes sino, de manera particular, potenciar el desarrollo del pensamiento crítico en ellos. Los profesores son determinantes en la formación del pensamiento crítico en los estudiantes, esto exige que participen de manera consciente e intencionada en el desarrollo de sus propias habilidades de pensamiento crítico.

La formación del pensamiento crítico en los estudiantes es uno de los propósitos de la educación y, en particular, de la Didáctica de las Ciencias. Los resultados de investigación aquí presentados ponen el acento en la exploración de los posibles obstáculos de estudiantes de grados cuarto y quinto de educación básica primaria frente al pensamiento crítico. Profundiza en el estudio de tres dimensiones: solución de problemas, argumentación y metacognición, las cuales son centrales en la formación del pensamiento crítico de los estudiantes. La interacción entre estas tres dimensiones del pensamiento permite comprensiones más profundas acerca del desempeño de los estudiantes y brinda posibilidades para identificar posibles obstáculos que se esgriman como amenazas en el interior de cada una de las dimensiones analizadas o entre las interacciones entre ellas, y que, a su vez, permitan orientar acciones educativas en función de lograr mayores desarrollos del pensamiento crítico de los estudiantes.

Parece claro que hablar de pensamiento crítico en la actualidad dista mucho del seguimiento de normas y prescripciones; es un desapego ante métodos estandarizados; va más allá del salón de clase, y se ha relacionado con la reflexión, la resolución de problemas, la toma de decisiones, entre otras habilidades. También se relaciona con las actitudes, los valores y los intereses de las personas. En el marco de este tipo de formación, el estudiante debe ser activo frente al aprendizaje. Esta actividad ha sido caracterizada de muy diversas maneras.

Formar pensamiento crítico en los estudiantes exige, entonces, la discusión de aspectos centrales como: a) reconocimiento de la estructura cognitiva del 
sujeto, su historia, experiencia, pensamiento; b) fomento de relaciones entre la ciencia y su conocimiento público, sobre las relaciones entre ciencia, tecnología, sociedad, ambiente y desarrollo; c) valoración de la dinámica propia de la ciencia, su funcionamiento interno y externo que la hacen funcional según el contexto y las condiciones de enseñanza y aprendizaje; d) priorización de los procesos conscientes y autorregulados en el aprendizaje de las ciencias, como mecanismo que permita profundizar y comprender cómo aprende el sujeto, para articularlo a procesos de enseñanza; e) reconocimiento de la escuela como escenario que brinda la posibilidad de acceder al conocimiento y donde recoge aportes fundamentales para construir y reconstruir el conocimiento de manera consciente e intencionada.

Una vez expuestos los aspectos conceptuales acerca de la importancia del desarrollo del pensamiento crítico en el aula de ciencias, nos dedicaremos a plantear las principales conclusiones derivadas de la investigación realizada sobre las categorías argumentación, pensamiento crítico y metacognición, como constituyentes del pensamiento crítico en estudiantes de educación básica primaria. El lector podrá encontrar detalles del proceso de investigación en Tamayo, Zona y Loaiza (2014).

\section{Metodología}

La investigación siguió un enfoque mixto en el que se estudió el problema del pensamiento crítico en procesos de enseñanza y aprendizaje de las ciencias naturales en aulas de educación básica primaria. Se exploraron las categorías argumentación, solución de problemas y metacognición en los estudiantes, con el propósito de comprender relaciones entre estas categorías como constituyentes del pensamiento crítico en ellos. Asimismo, se des- cribieron las concepciones de maestros acerca del pensamiento crítico. Dado el propósito de la investigación, se espera la generación de conocimiento en torno a la categoría central pensamiento crítico, a la cual aportarán los actores inmersos (estudiantes y maestros). La población de niños y niñas participantes en esta investigación fue de 2240 de 56 instituciones educativas (publicas) de la ciudad de Manizales, y cursaban los grados cuarto y quinto de primaria. La muestra analizada corresponde a 224 estudiantes y 5 maestros. La investigación se realizó en tres momentos:

1. Diseño de una propuesta didáctica para el desarrollo de pensamiento crítico. Se elaboraron diferentes actividades de aula a partir de las tres categorías centrales: argumentación, solución de problemas y metacognición (anexo 1). En esta fase se incluye el proceso de formación de profesores orientado al desarrollo del pensamiento crítico en los estudiantes.

2. Aplicación de la propuesta de enseñanza.

3. Descripción comprensiva del pensamiento crítico en estudiantes de básica primaria a partir de la relación entre argumentación, solución de problemas y metacognición.

Tanto en lo referido a los análisis de la información proveniente de los niños como de los maestros, y debido al carácter mixto de la investigación, se realizaron análisis cuantitativos y cualitativos que permitieron mejores comprensiones de las diferentes categorías estudiadas y las relaciones entre ellas. Las tablas de distribución de frecuencias permitieron identificar las tendencias más y menos relevantes que asignan niños y maestros al pensamiento crítico. Las redes semánticas facilitaron establecer relaciones entre las cate- 
gorías centrales, identificar categorías emergentes y lograr mejores comprensiones de cada una de las categorías estudiadas.

Las diferentes actividades de aula presentadas partían de un problema de ciencias, el cual debía ser resuelto experimentalmente o mediante el empleo de diferentes estrategias seleccionadas por los estudiantes. Los materiales requeridos para la realización de las actividades fueron suministrados por los investigadores, quienes previamente realizaron los procesos de validación y pilotaje de estas. Asimismo, adelantaron un proceso de formación docente a 77 profesores asistentes, los cuales se encargaron de realizar, en conjunto con los profesores titulares de las 56 instituciones educativas, las diferentes actividades propuestas orientadas a caracterizar el pensamiento crítico en estudiantes. En la tabla 1 describimos los niveles para evaluar la calidad de la argumentación, de la solución de problemas y las subcategorías utilizadas para evaluar la metacognición.

Tabla 1. Niveles y categorías de análisis

\begin{tabular}{ll}
\hline Nivel 1 & $\begin{array}{l}\text { Comprende los argumentos que son una descripción simple de la vivencia (Van Dijk \& } \\
\text { Kintsch, 1983). }\end{array}$ \\
\hline Nivel 2 & $\begin{array}{l}\text { Comprende argumentos en los que se identificaron claridad de los datos (data) y } \\
\text { conclusión (Claim). }\end{array}$ \\
\hline Nivel 3 & $\begin{array}{l}\text { Son argumentos constituidos por datos, con conclusiones y una justificación (warrant), y } \\
\text { sin cualificador o modalizador. }\end{array}$ \\
\hline Nivel 4. & $\begin{array}{l}\text { Comprende argumentos constituidos por datos, conclusiones, justificaciones (warrants) } \\
\text { haciendo uso de cualificadores (qualifiers) o respaldo teórico (backing), y sin }\end{array}$ \\
\hline Nivel 5. & Comprende argumentos con conclusión y un contraargumento (rebuttal). lgual. \\
\hline Nivel 6. & Comprende argumentos completos con más de un contraargumento (rebuttal). \\
\hline
\end{tabular}

Solución de problemas

\begin{tabular}{ll}
\hline Nivel 1 & \multicolumn{1}{c}{ Solución de problemas } \\
\hline Nivel 2 & $\begin{array}{l}\text { Redescripción de la experiencia, enuncia el problema y describe el experimento según } \\
\text { sus observaciones o utiliza datos de las instrucciones para justificar sus respuestas. } \\
\text { anteriormente, utiliza opiniones, describe lo que sintió durante las experiencias o utiliza } \\
\text { analogías. }\end{array}$ \\
\hline Nivel 3 & $\begin{array}{l}\text { Identificación de una o dos variables, en este nivel se reconocen las variables sin realizar } \\
\text { algún tipo de relación entre ellas. }\end{array}$ \\
\hline Nivel 4 & $\begin{array}{l}\text { Resolución del problema de manera inadecuada identificando y relacionando variables y } \\
\text { justificando o no dichas relaciones. }\end{array}$ \\
\hline Nivel 5 & $\begin{array}{l}\text { Resolución de problema de manera adecuada identificando, relacionando variables y } \\
\text { justificando o no dichas relaciones. }\end{array}$ \\
\hline
\end{tabular}

Metacognición

La evaluación de la categoría metacognición se realizó a partir de tres subcategorías: tipo de conocimiento, conciencia metacognitiva y regulación (Tamayo, 2006). Los análisis se realizaron teniendo en cuenta las expresiones nucleares (Chomsky, 2004) elaboradas por los estudiantes y los profesores.

Dado el alcance de este documento, nos dedicaremos a continuación a presentar las principales conclusiones encontradas para las categorías argu- 
mentación, metacognición y solución de problemas, sin entrar en detalles de naturaleza metodológica acerca de cómo se realizaron los diferentes análisis y discusiones (para mayor información, ver Tamayo, Zona y Loaiza, 2014).

\section{Acerca de las categorías argumentación, metacognición y solución de problemas}

Diseño de ambientes de enseñanza y aprendizaje orientados al desarrollo de habilidades argumentativas en los estudiantes. Se requiere que el actuar del maestro en las aulas de clase esté orientado a que el estudiante identifique y diferencie con claridad los datos de las conclusiones, las justificaciones, los respaldos teóricos y los contraargumentos. De igual manera, es necesario que tanto profesores como estudiantes comprendan que la argumentación en ciencias es condición sine qua non para el logro de comprensiones profundas de lo estudiado y, asimismo, es la actividad central del trabajo científico.

- En la práctica cotidiana de la argumentación en el aula intervienen diferentes dimensiones, las cuales interactúan de manera interdependiente. La argumentación involucra procesos cognitivos, interactivos y dialógicos, en torno a temas específicos y en el marco de contextos institucionales y culturales determinados. Algunas de las dimensiones que se deben tener en cuenta son: el individuo con sus propias capacidades cognitivas y comunicativas, los interlocutores con su estatus e intenciones, el tópico discutido, las herramientas usadas y el contexto sociocultural (Muller, Perret-Clermont, Tartas \& lannaccone, 2009). En cuanto a la dimensión intrapersonal de la argumentación nos referimos a las herramientas del pensamiento, a nivel individual, requeridas para participar en los procesos argumentativos; esto implica comprender los prerrequisitos cognitivos que le permiten a los sujetos participar en los procesos argumentativos, lo cual incluye la dimensión afectiva y las maneras como se relacionan los sujetos con los temas de discusión y con las herramientas de mediación en la construcción de los argumentos. En cuanto a la dimensión dialógica e interpersonal, se debe reconocer que la argumentación es una actividad que requiere, en la mayoría de los casos, la interacción con los otros en torno a un tema o problema específico. Comprender la complejidad de la actividad argumentativa exige descentrarnos del sujeto y del argumento que él produce en un momento determinado. En términos de los autores, la argumentación es un tipo particular de diálogo que contribuye a que los individuos adquieran aprendizajes en temas específicos, así como en lo relacionado con prácticas culturales; los contextos socioculturales específicos en los cuales ocurre la argumentación orientan, limitan y contribuyen a la forma como se presente finalmente el argumento, en este sentido la argumentación es siempre situada, se da siempre en un lugar y tiempo específicos. Asimismo, nos permite comprender las características del interlocutor. En cuanto al tópico específico en el cual se realiza 
la actividad argumentativa, es claro que este puede facilitar o limitar los procesos y productos argumentativos.

- En cuanto a los temas específicos, el desarrollo de habilidades argumentativas en el aula exige, de parte del maestro, la planeación detallada de los procesos de transposición didáctica. Esto exige, por parte del maestro, reflexiones en torno a las transformaciones permitidas entre el objeto de saber, el de enseñanza y el de aprendizaje. A su vez, este componente conceptual es sensible a aspectos, ontológicos, cognitivo-lingüísticos y motivacionales de los participantes en el proceso argumentativo.

- La experiencia de los sujetos, su aspecto ontológico, determina el desempeño argumentativo. La experiencia argumentativa, de discusión, de confrontación, en la que han participado las personas a lo largo de su vida, constituyen los presaberes o los modelos argumentativos que ponen en ejercicio los estudiantes en un momento determinado. Vinculado con el aspecto ontológico, las habilidades cognitivo-lingüísticas de los estudiantes hacen referencia a los usos del lenguaje y a aquellas habilidades cognitivas que ejercitan los estudiantes, tales como el análisis, la síntesis, la teorización y la conceptualización, entre otras, durante el proceso de argumentación.

- Además del lenguaje oral-escrito presente en las aulas de clase, encontramos que los procesos de enseñanza y aprendizaje, y con ellos el desarrollo de habilidades argumentativas, tienen como mediadores otros lenguajes (Lemke 1999, Martins 2000, 2001, Scott 2000, Jewitt 2000, Mortimer 1998, Tamayo et. al. 201 1); se hace referencia, por ejemplo, al lenguaje oral, escrito, gestual, gráfico y corporal, entre otros.

- Dentro de los aspectos cognitivos en el proceso de elaboración de argumentos uno es de especial importancia: el conocimiento y control que se tiene sobre los propios procesos del pensamiento, conocido como metacognición, determinante en el logro de aprendizajes profundos por parte de los estudiantes. El vínculo entre metacognición y argumentación es sugerente tanto en el ámbito teórico como en el plano de la acción en las aulas de clase, de tal manera que regular de forma consciente e intencionada los desempeños argumentativos es en uno de los fines de la enseñanza y el aprendizaje de las ciencias. Cuando un estudiante identifica sus fortalezas y debilidades al participar en espacios argumentativos y, a su vez, tiene la capacidad para monitorear, evaluar y regular sus desempeños, tiene conciencia acerca de sus capacidades o competencias argumentativas. Este, sin duda, se constituye en uno de los propósitos centrales en la enseñanza.

- La argumentación, como todo actuar de los seres humanos, es sensible a las motivaciones y a los afectos. De manera análoga a lo 
mencionado acerca de la metacognición, la metaafectividad se refiere al conocimiento y control de los propios procesos afectivos. Conocer y regular nuestras expresiones de afectividad en el proceso de elaborar argumentos nos acerca, sin duda, a un mejor conocimiento de nuestra cognición y, de manera específica, de los procesos afectivos involucrados en el aprendizaje en dominios específicos del conocimiento.

- Los diferentes análisis de las categorías de la metacognición: tipo de conocimiento, conciencia metacognitiva y regulación, en los tres momentos de la investigación, no mostraron resultados satisfactorios en cuanto a la cualificación de estas habilidades por parte de los estudiantes a medida que se realizan las diferentes actividades de aula. Encontramos una tendencia general en los estudiantes a disminuir su inseguridad en las respuestas dadas a las diferentes preguntas planteadas a lo largo de los tres momentos de análisis. Asimismo, en el momento 2 se observó un incremento importante en la percepción de seguridad que tienen los estudiantes frente a las respuestas dadas. Este incremento global en la seguridad de los estudiantes a lo largo del desarrollo de las diferentes actividades propuestas estuvo acompañado del fortalecimiento de la subcategoría conciencia/experimentación, lo cual significaría que la seguridad que gana el estudiante está anclada de manera específica a la realización cuidadosa del experimento que se le propone. En otras palabras, seguir los pasos sugeridos por la actividad experimental da seguridad a los estudiantes acerca de las observaciones realizadas y de las explicaciones ofrecidas a las diferentes situaciones presentadas.

- En cuanto a la resolución de problemas los estudiantes muestran cierta tendencia al empleo de niveles más exigentes a medida que la intervención de aula avanza. Este movimiento hacia niveles resolutivos de problemas de mayor exigencia para los estudiantes se deriva posiblemente del trabajo intencionado realizado por los profesores en función del desarrollo de ciertas habilidades en la resolución de problemas de los estudiantes, a partir del conjunto de actividades desarrolladas a lo largo de la intervención didáctica.

- Los textos ubicados en el nivel 1 de resolución de problemas representan la re-descripción de la situación dada en los diferentes experimentos y actividades de aula. Su característica central es el empleo de las mismas expresiones utilizadas en la situación presentada, de tal manera que los estudiantes terminan describiendo lo que observaron. El nivel 2 de resolución de problemas comprende redescripciones de la experiencia de manera libre: "ha realizado la experiencia anteriormente", "utiliza opiniones", "describe lo que sintió durante las experiencias o utiliza analogías". Se destaca de las redescripciones libres antes descritas las múltiples estrategias personales que se emplean para resolver el problema, unas enfocadas en procesos memorísticos al plantear el desarrollo de la experiencia previamente, otras 
que realizan relaciones como es el uso de analogías y otras que se basan en creencias y opiniones.

- Las explicaciones, los respaldos teóricos y, en última instancia, el uso y relación de variables pueden ser empleadas por los estudiantes para referirse a las situaciones presentadas, insinuando comprensiones superficiales de los fenómenos. En este sentido la aprehensión de la situación a la que alude el problema presentado a los estudiantes está mediada por la apariencia externa del fenómeno. Los estudiantes identifican con cierta facilidad los datos proporcionados en la situación, se refieren a la conclusión, logran presentar justificaciones derivadas de la experiencia directa, pero no respaldan teóricamente estas justificaciones. La ausencia de respaldos teóricos sólidos es, sin duda, un aspecto que se logra con la escolarización, sin embargo, es la principal responsable de respaldos teóricos débiles. En tal sentido, la enseñanza debe orientar esfuerzos de manera intencionada para conseguir que los estudiantes no solo aprehendan el fenómeno en su forma, sino, además, y lo que es más importante, que le permitan referirse a él en el plano abstracto.

- Las respuestas que dan los estudiantes a los problemas en esta investigación se ubican en los tres primeros niveles, en el momento 1 con un $84,5 \%$; en el momento 2 con $86,6 \%$ y con un $68,8 \%$ en el momento 3. Esto nos permite afirmar que los estudiantes tienen comprensiones superficiales de los fenómenos presentados. Se requieren intervenciones didácticas que permitan desarrollar habilidades (cognitivas, cognoscitivas, metacognitivas), actitudes y herramientas heurísticas en la resolución de problemas.

- Con las intervenciones en el aula de ciencias se desarrollan diferentes facultades en nuestros estudiantes, entre ellas: en primer lugar, la independencia cognoscitiva, que se define como la capacidad para determinar elementos cruciales en objetos, fenómenos y procesos; organizar los materiales debatir y criticar científicamente sus propias tesis y las de los demás, asimilar y aplicar adecuadamente los nuevos conocimientos y habilidades en la resolución de problemas. En segundo lugar el desarrollo de actitudes es uno de los componentes en la enseñanza por resolución de situaciones problémicas, ya que son las actitudes de los individuos las que muestran su comportamiento ante los objetos sociales, el conocimiento y la resolución de problemas. 
Acerca de las concepciones de los profesores sobre pensamiento crítico

En cuanto al pensamiento crítico en los profesores investigados podemos concluir:

- Los maestros se refieren al pensamiento crítico desde diferentes perspectivas teóricas. Al igual que en la literatura presentada a lo largo de este texto, las concepciones de los profesores sobre este concepto son difusas y, en gran medida, están orientadas desde el sentido común acerca del pensamiento crítico y sobre cómo se desarrolla en las aulas de clase. Es necesario establecer relaciones entre pensamiento crítico y la dinámica interna que lo potencia, es decir, articular su desarrollo a procesos cognitivos conscientes, a promover espacios autorreguladores que permitan hacer más eficiente su potenciación y a brindar herramientas de apoyo para la planeación, monitoreo y evaluación de los procesos conducentes a su desarrollo.

- El contexto es considerado como un detonador del pensamiento crítico. Es asumido como un espacio donde se aplican y construyen conocimientos; por consiguiente, una implicación didáctica consiste en enseñar y aprender desde diversos contextos y espacios; es decir, los laboratorios, museos, zoológicos, espacios virtuales, entre otros, pueden conducir a desarrollar habilidades y actitudes que constituyen el pensamiento crítico.

- Se recomienda, antes de incorporar programas para potenciar el pensamiento crítico, conocer las concepciones de los maestros, saber sus opiniones acerca del pensamiento crítico, con el propósito de identificar posibles obstáculos que dificultarían su desarrollo. Asimismo, conviene reconocer la falta de consenso sobre este concepto, con las consecuentes ventajas y desventajas que esto puede ocasionar. La caracterización realizada con maestros de grados tercero, cuarto y quinto de básica primaria de la Escuela Normal Superior de Caldas, nos permite señalar de manera específica, los siguientes aspectos: a) la indagación se constituye en una categoría fundamental en la constitución de pensamiento crítico de los maestros y b) en el grupo de profesores investigados el pensamiento crítico es sensible al contexto.

En lo concerniente a las categorías reflexión, curiosidad y persistencia, son consideradas dentro de las concepciones aunque de manera superficial; lo que sugiere explorar y profundizar las causas e implicaciones en posteriores estudios. De igual manera, las categorías motivación y metodología son de gran relevancia en cuanto a las habilidades que debe poseer un maestro con pensamiento crítico, categorías que en las perspectivas teóricas presentan poca profundidad en sus estudios y que se sugiere incluirlas como habilidades propias de un maestro que potencia el desarrollo del pensamiento crítico en los estudiantes.

- Los maestros de básica primaria investigados emplean estrategias de orden metodológico para potenciar pensamiento crítico, centradas en la realización de consultas, en actividades de lectura. Consideran que el desarrollo del pensamiento crítico se puede potenciar en el aula 
haciendo énfasis en aspectos de naturaleza metodológica, dejando a un lado aspectos de naturaleza conceptual, determinantes en cuanto a la variedad de perspectivas teóricas que se han ocupado del estudio del pensamiento crítico y en cuanto a las orientaciones metodológicas que de ellos se pueden derivar. Este marcado énfasis en aspectos de naturaleza metodológica puede llevar a convertir los pocos espacios destinados al logro del pensamiento crítico en espacios en los que se privilegia una actividad poco reflexionada y poco fundamentada conceptualmente por los profesores, con el consecuente riesgo de llegar a considerar el pensamiento crítico como una estrategia de trabajo docente, más que como un constructo teórico orientador de acciones de enseñanza, actitudes de vida, formas de pensamiento y de actuación en ámbitos generales y de dominios específicos del conocimiento.

- Los maestros de básica primaria no conocen en profundidad qué es el pensamiento crítico, las estrategias que plantean son pocas y no están soportadas teóricamente. Su acercamiento al concepto, tanto teórico como metodológico, proviene de reflexiones ubicadas en el ámbito del sentido común, con el consecuente desconocimiento casi total de las diferentes perspectivas teóricas que históricamente se han construido acerca de este concepto. Asimismo, se observó el desconocimiento de estrategias de enseñanza exitosas y no exitosas, que se han aplicado en diferentes contextos educativos. Puede afirmarse que la actuación de los profesores en torno al desarrollo y potenciación del pensamiento crítico sigue los principios propios del ensayo y del error.

- Las dificultades que plantean los maestros de básica primaria se centran en cinco categorías: desarrollo de habilidades mentales, aspectos motivacionales, poco apoyo familiar, conocimiento superficial, políticas institucionales. Sin embargo, los maestros no ponen en manifiesto dificultades que vinculen su formación académica, con el fin de mejorar los procesos de enseñanza y aprendizaje en las aulas de clase. Las dificultades señaladas por los profesores se centran en el desempeño de los estudiantes y no en las actuaciones de los profesores. Es decir, la debilidad en cuanto al pensamiento crítico en los estudiantes se debe, según los profesores, a las condiciones de los propios estudiantes y no a las de los docentes.

- Ausencia importante de procesos que apoyen la formación de los profesores acerca del desarrollo y potenciación del pensamiento crítico en las aulas de clase. Somos los profesores, y la escuela como institución privilegiada en torno a la formación de los sujetos, quienes tenemos, o deberíamos tener, mayor claridad teórica y metodológica acerca de la formación del pensamiento crítico, particularmente en dominios específicos del conocimiento. 
- Se requieren propuestas y programas orientados a articular horizontalmente esfuerzos de diferente naturaleza en función de potenciar el pensamiento crítico en la escuela. En pocas palabras, formar en pensamiento crítico no es solo un desafío de la escuela como institución.

\section{Referencias bibliográficas}

Chomsky, N. (2004). Estructuras sintácticas. México: Siglo Veintiuno.

Dagher, Z.R. (1994). Does the use of analogies contribute to conceptual change? Science Education $78(6), 601-614$.

Erduran, S. (2008). Methodological foundations in the study of argumentation in science classrooms. In: Erduran, S. \& Jiménez-Aleixandre, M. P. (Eds.) Argumentation in Science Education. Perspectives from Classroom-Based Research. (pp. 47-69). Dordrecht: Springer.

Erduran, S. \& Jiménez-Aleixandre, M. P. (2008). Argumentation in Science Education. Perspectives from Classroom-Based Research. Dordrecht: Springer.

Jewitt, C. (2000). Formas de comunicación en la clase de ciencias. En: Enseñar ciencias: construcción de significados i comunicación multimodal. Documento no publicado. Universidad de Barcelona.

Jiménez-Aleixandre, M. P. (2008). Designing argumentation learning environments. In: M. P. Jiménez-Aleixandre \& S. Erduran (Eds.). Argumentation in science education. Perspectives from classroom-based research (pp. 91-115). Dordrecht: Springer.

Jiménez-Aleixandre, M. y Erduran, S. (2008). Argumentation Science Education: An Overview. En: M. P. Jiménez-Aleixandre y S. Erduran (eds.). Argumentation in science education. Perspectives from classroom-based research (pp. 3-27). Dordrecht: Springer.

Justi, R. (2006). La enseñanza de ciencias basada en la elaboración de modelos. Enseñanza de las Ciencias 24(2), 173-184.

Lemke, J. L. (1999). Multiplying meaning: visual and verbal semiotics in scientific text. En: J. R. Martin y R. Veel (Eds.). Scientific discourse. Londres: Longman. Lenguaje y Educación.

Mortimer, E. F. (1998). La tensió entre deàleg y mobolèg en la interacció profesor-alumne a I'aula de cièncie. En: Ideas para una cultura científica. Barcelona: Museo de la Ciencia.

Martins, I. (2000). Onda u particula: argumentacao e retòrica na aprendizagem da natureza da luz. Trabalho apresentado e publicaron as Atas do VII Encontro de pesquisa em ensino de física, Florianópolis, SC, Brasil, 27-31 de Mayo.

Martins, I. (2001). Analisi retòrica i recerca en educación científica. Seminario de reserca. Universidad de Barcelona. Documento no publicado.

Muller, N., Perret-Clermont, A., Tartas, V. \& lannaccone, A. (2009). Psychosocial processes in argumentation. In: N. Muller \& A. Perret-Clermont (Eds.). Argumentation and education. Theoretical foundations and practice. (pp. 67-90). Breinigsville, PA: Springer 
Palma, H. (2004). Metáforas en la evolución de las ciencias. Buenos Aires: Jorge Baudino Editores.

Porlán, R.; Rivero, A. y Martín Del Pozo, R. (1998). Conocimiento profesional y epistemología de los profesores, II: Estudios empíricos y conclusiones. Enseñanza de las Ciencias 16(2), 271-288.

Pfundt, H. y Duit, R. (1991). Bibliography students' alternative frameworks and science education. 3a. ed. Institute for Science Education. IPN: Kiel.

Pfundt, H. y Duit, R. (1993). Bibliography: Students' alternative frame works and science education. Kiel: University of Kiel.

Ruiz, F.; Márquez, C. y Tamayo O. E. (2014). Cambio en las concepciones de los docentes sobre la argumentación y su desarrollo en clase de ciencias. Enseñanza de las Ciencias 32(3), 53-70.

Scott, P. (2000). Teacher talk and meaning-making in science classrooms. En: Enseñar ciencias: construcción de significados i comunicación multimodal. Universidad de Barcelona. Documento no publicado.

Tamayo, O. (2006). La Metacognición en los modelos para la enseñanza y el aprendizaje de las ciencias. En: Los bordes de la pedagogía: del modelo a la ruptura (pp 275-306). Universidad Pedagógica Nacional. Bogotá: Net Educativa Editorial

Tamayo, O. (2009). Didáctica de las ciencias: La evolución conceptual en la enseñanza y el aprendizaje de las ciencias. Manizales: Ed. Universidad de Caldas.

Tamayo, O., Vasco, C. E., Suárez, M. M., Quiceno, H., García, L. I. y Giraldo, A. (2011). La clase multimodal y la formación y evolución de conceptos científicos a través del uso de tecnologías de la información y la comunicación. Manizales: Universidad Autónoma de Manizales.

Tamayo, A.O. E., Zona, R. y Loaiza, Y. E. (2014). Pensamiento crítico en el aula de ciencias. Manizales: Universidad de Caldas.

Toulmin, S. (1972). La comprensión humana. El uso colectivo y la evolución de los conceptos. Madrid: Alianza Editorial.

Van Dijk, T. \& Kintsch, W. (1983). Strategies of discourse comprehension. Nueva York: Academic Press.

Vélez Gutiérrez, Carlos Fernando. (2013). “Una reflexión interdisciplinar sobre el pensamiento crítico". Revista Latinoamericana de Estudios Educativos. No. 2, Vol. 9, pp. 11-39. Manizales: Universidad de Caldas. 
Anexo 1.

\section{Instrumento 1}

Nombre

Edad Grado

\section{Situación problema}

Materiales: papel, agua y mechero o vela, hilo.

Pon el papel en contacto con la llama de la vela. A partir de lo observado responde las siguientes preguntas:

1. ¿̇Qué crees que sucederá cuando pones el papel en contacto con la llama? Justifica tu respuesta.

2. ¿̇Por qué crees que se enciende el papel?

A continuación realiza la experiencia que te propone el profesor.

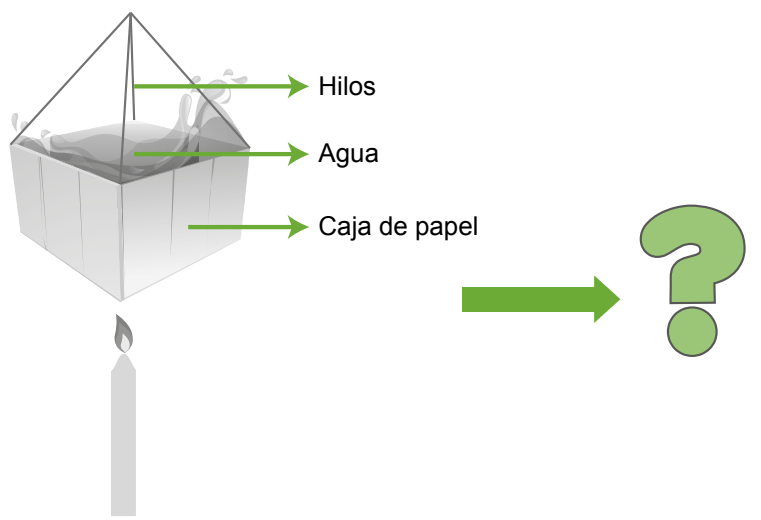

En relación con la figura responde la siguiente pregunta

3. ¿Qué crees que sucederá cuando la llama de la vela entra en contacto con la caja de papel que contiene agua? Justifica tu respuesta. 
4. Una vez realizada la experiencia, ¿̇qué sucedió cuando la llama entró en contacto con la caja que contiene agua? Justifica tu respuesta.

5. ¿QQué crees que sucedería si en lugar de agua ponemos arena en la caja de papel? Justifica tu respuesta.

6. ¿Cuáles crees que son algunos de los usos que hacemos cotidianamente de esta propiedad que tiene el agua?

7. ¿Crees que tus respuestas son correctas o incorrectas? Justifica tu respuesta.

\section{Discusión}

Una vez contestadas las preguntas antes planteadas, reúnete en grupos de 5 estudiantes para realizar las siguientes actividades:

Cada estudiante leerá sus respuestas a los demás compañeros. Una vez todos hayan leído, respondan las siguientes preguntas:

8. Justifiquen detalladamente lo sucedido en cada una de las situaciones:

8.1 Papel en contacto con la llama:

8.2 Caja con agua en contacto con la llama: 
9. Cuando realizaste el trabajo en grupos, ¿̇qué nuevos aprendizajes lograste?

10. ¿2Qué diferencias encuentras entre lo que pensabas antes y después de la experiencia?

11. ¿̇Cómo y cuándo te das cuenta de que tus pensamientos sobre el experimento son correctos o incorrectos?

12. ¿Cuándo se te presenta un experimento como el realizado hoy, cómo llegas a resolver el problema planteado?

Gracias por tu colaboración 
